\title{
GATA-3 promotes Th2 responses through three different mechanisms: induction of Th2 cytokine production, selective growth of Th2 cells and inhibition of Th1 cell-specific factors
}

\author{
Jinfang Zhu ${ }^{1}$, Hidehiro Yamane ${ }^{1}$, Javier Cote-Sierra ${ }^{1}$, Liying Guo ${ }^{1}$, William E Paul ${ }^{1}$ \\ ${ }^{1}$ Laboratory of Immunology, National Institute of Allergy and Infectious Diseases, National Institutes of Health, Bethesda, MD 20892, \\ USA
}

Naïve CD4 T cells can differentiate into at least two different types of Thelpers, Th1 and Th2 cells. Th2 cells, capable of producing IL-4, IL-5 and IL-13, are involved in humoral immunity against extracellular pathogens and in the induction of asthma and other allergic diseases. In this review, we summarize recent reports regarding the transcription factors involved in Th2 differentiation and cell expansion, including Stat5, Gfi-1 and GATA-3. Stat5 activation is necessary and sufficient for IL-2-mediated function in Th2 differentiation. Enhanced Stat5 signaling induces Th2 differentiation independent of IL-4 signaling; although it does not up-regulate GATA-3 expression, it does require the presence of GATA-3 for its action. Gfi-1, induced by IL-4, promotes the expansion of GATA-3-expressing cells. Analysis of conditional Gata 3 knockout mice confirmed the critical role of GATA-3 in Th2 cell differentiation (both IL-4 dependent and IL-4 independent) and in Th2 cell proliferation and also showed the importance of basal GATA-3 expression in inhibiting Th1 differentiation.

Cell Research (2006) 16:3-10. doi:10.1038/sj.cr.7310002; published online 16 January 2006

Keywords: GATA-3, Stat5, Gfi-1, Th2 cell differentiation, cell expansion, transcription factors

\section{Introduction}

Upon antigen stimulation of their $\mathrm{T}$ cell receptors by antigen presenting cells (APCs), naïve CD4+ T cells can differentiate towards at least two different types of Thelpers, Th1 and Th2 cells [see recent Reviews 1, 2]. These cells play important roles in adaptive immunity. Th1 cells produce IFN $\gamma$ and are involved in immunity against intracellular pathogens. Th2 cells can produce IL-4, IL-5 and IL-13 and are involved in humoral immunity against extracellular pathogens. Th1 and Th2 cells are also involved in the induction or maintenance of human diseases. Th1 cells are linked to organ-specific autoimmunity and Th2 cells play critical roles in asthma and other allergic diseases.

Although peptide affinity for TCR, concentration of

Correspondence: Jin Fang Zhu

Tel: 301-402-6662; Fax: 301-480-7352;

E-mail:.jfzhu@niaid.nih.gov peptide and the particular co-stimulatory molecules involved play important roles in determining the fate of T cell differentiation, the cytokine milieu is the most important factor for this process. Strikingly, the stimulatory cytokines are the effector cytokines themselves. Thus, IFN $\gamma$ induces Th1 differentiation and IL-4 induces Th2 differentiation. During Th1 differentiation, IFN $\gamma$, possibly made by NK, NKT or memory $\mathrm{T}$ cells, induces T-bet, the master regulator of Th1 cells, in activated CD4 T cells [3-6]. Up-regulation of T-bet can either promote IFN $\gamma$ production directly or indirectly by up-regulating IL-12R $\beta 2$ expression [7]. IL12 made by APC selectively enhances the growth of T-bet expressing cells [8]. IL-12 mediated Stat 4 activation can also induce IFN $\gamma$ production independent of T-bet. The IFN $\gamma /$ T-bet and IL-12/Stat4 pathways crosstalk and synergize with each other under most circumstances. For Th2 differentiation, Stat 6 activation is necessary and sufficient to transduce IL-4 signaling, at least in vitro [9-11]. IL-2 mediated Stat 5 activation is also critical for this process $[12$, 13]. In this review, many recent reports by our laboratory 
regarding the molecules involved in Th2 differentiation and cell expansion will be summarized.

\section{IL-2 and Stat5 in Th2 differentiation}

It was reported in 1991 that IL-2 is important for Th2 differentiation $[14,15]$; however, very little attention was drawn to this signaling pathway in the differentiation process since it was thought that IL-2 might simply serve as a $\mathrm{T}$ cell growth factor. Recently, Cote-Sierra et al have shown that IL-2 is not required for T cell proliferation and/or survival if IL-4 is added in the culture [12]. Thus, when naïve 5C.C7 CD4 T cells, derived from $\mathrm{Rag} 2^{-/-} \mathrm{TCR}$ transgenic mice, in which the TCR is specific for pigeon cytochrome $\mathrm{C}$ (PCC), were primed under Th2 conditions in vitro (peptide or anti-CD3/anti-CD28 plus IL-4, anti-IL-12 and anti-IFN $\gamma$ ), the proliferative rate, as judged by CFSE dilution, of the cells primed in the absence of IL-2 signaling (through the addition of anti-IL-2 and anti-IL-2R $\alpha$ ) was similar to that of the cells primed in the presence of IL-2, as was the total cell yield. However, IL-4 production, measured by cytokine intracellular staining after anti-CD3/ anti-CD28 re-stimulation, was dramatically reduced. In addition, the early IL-4 production driven by low peptide stimulation also depends on the IL-2 signaling [16].

Stat5 is one of the key downstream molecules mediating IL-2 signaling [17]. To test whether the IL-2's role in Th2 differentiation is through Stat5 activation, Stat5a-deficient CD4 T cells were used for Th2 priming. Even in the presence of IL-2, these Stat5a-deficient CD4 T cells failed to undergo $\mathrm{Th} 2$ differentiation implying that Stat5a activation is required for this differentiation process. A constitutively active form of Stat5a (STAT5A1*6) [18] was introduced by retroviral infection into CD4 T cells during Th2 priming; it was found that expression of STAT5A $1 * 6$ fully rescued the IL-4 producing capacity of the cells primed under Th2 conditions in the absence of IL-2, suggesting that Stat5 activation is sufficient to transduce IL-2 signaling for Th2 priming [12]. Many other cytokines including IL-4, IL-7, IL-9 and IL-15 also activate Stat5 [17]; however, the degree of Stat5 tyrosine phosphorylation in these CD4 T cells was greatest in response to IL-2 [12]. In addition, in Stat5adeficient cells, IL-2 can still activate Stat5b. Therefore, weak Stat5 activation remained when cells were cultured in the absence of IL-2 or when Stat5a-deficient cells were cultured in the presence of IL-2, but such weak activation appears to be insufficient for Th2 differentiation. These results suggest that $\mathrm{Th} 2$ differentiation requires robust Stat5 activation, whereas $\mathrm{T}$ cell proliferation remains intact with low levels of Stat5 activation.

STAT5A1*6-expressing cells can acquire IL-4-producing capacity even when cultured under Th1 conditions (peptide or anti-CD3/anti-CD28 plus IL-12 and anti-IL-4) in the presence of IL-2 [13]. Thus, the Stat5-mediated induction of IL-4-producing capacity is IL-4 independent. Such induction was also observed in Stat6 deficient or in IL-4R $\alpha$ deficient CD4 T cells [13]. The increased Stat5 signaling does not cause general up-regulation of cytokineproducing capacity since IFN $\gamma$ production was not detected in STAT5A1*6-expressing Th2 cells. To further test the specificity of Stat5 effect, CD4 T cells were primed under ThN conditions (non-polarized Th conditions, peptide or anti-CD3/anti-CD28 plus anti-IL-4, anti-IL-12 and antiIFN $\gamma$ ), after which neither IFN $\gamma$ nor IL-4 is produced upon re-stimulation. The introduction of STAT5A $1 * 6$ under such conditions caused a large percentage of the cells capable of making IL-4 with minor effect on IFN $\gamma$ production.

Th2 differentiation is usually associated with the upregulation of GATA-3 expression [19, 20]. Real-time PCR experiments suggest that the GATA-3 expression levels in STAT5A1*6-RV infected Th1 cells were not increased although these cells were capable of making IL-4 [13]. In addition, neutralizing IL-2 in Th2 culture did not affect the up-regulation of GATA-3 by IL-4 although IL-4 production was severely blocked [12]. Early IL-4 production (24hr after stimulation) in response to stimulation of naïve TCR transgenic cells is associated with the up-regulation of GATA-3 expression. Neutralization of IL-2 totally abolished the early IL-4 producing capacity but did not diminish GATA-3 up-regulation [16]. Thus, GATA-3 up-regulation and Stat5 activation may be regarded as two independent events in the induction of Th2 differentiation.

\section{Synergistic effect of GATA-3 and active Stat5 in IL-4 production}

Stat5 signaling appears to be independent of the up-regulation of GATA-3. Indeed, inhibiting Stat 5 activation does not affect the up-regulation of GATA-3 by IL-4 signaling $[12,16]$; enhanced Stat5 activation does not up-regulate GATA-3 expression [13]. In addition, the over-expression of GATA-3 in Th2 cells cannot rescue the defect in IL-4 production when Stat5 signaling is inhibited [12]. Since enhancing either of two distinct pathways can induce IL4 production, we studied whether there is a synergistic effect between them [13]. Cells co-expressing GATA-3 and STAT5A1*6, primed under Th1 or ThN conditions, had highest percentage of IL-4 producing cells as well as highest mean fluorescence intensity (MFI) of the IL-4producing cells, particularly when compared to the cells infected with GATA-3-RV or STAT5A1*6-RV alone in the same culture (Figure 1). This suggests that these two independent pathways can collaborate with each other in inducing IL-4 production. 


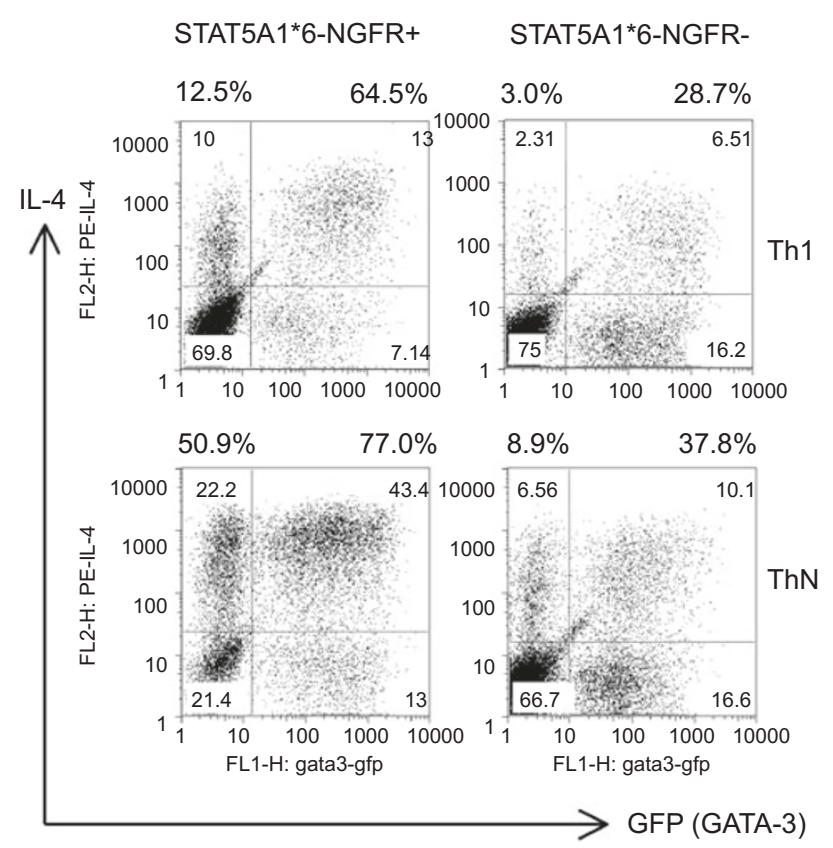

Figure 1 Co-infection with STAT5A1*6-NGFR-RV and GATA3-GFP-RV optimizes IL-4 production (adapted from Immunity 2003; 19: 739-48) [13]. Line 94 CD4 T cells were activated with T-depleted spleen cells as antigen presenting cells (APC) and pigeon cytochrome C (PCC) under ThN (anti-IL-4, anti-IFN $\gamma$ and anti-IL12) or Th1 conditions (IL-12 and anti-IL-4) for $40 \mathrm{~h}$ and co-infected with the STAT5A1*6-NGFR-RV and the GATA-3-GFP-RV. After an additional four-day culture under ThN or Th1 conditions, they were stimulated with PMA and ionomycin. Staining for IL-4 and for NGFR expression was carried out. Dot plots were gated on $\mathrm{NGFR}^{+}\left(\mathrm{STAT}^{2} \mathrm{~A} 1 * 6-\mathrm{NGFR}^{+}\right)$and NGFR${ }^{-}\left(\mathrm{STAT}^{2} \mathrm{~A} 1 * 6-\mathrm{NGFR}^{-}\right)$ populations.

The accessibility of regulatory sites to transcription factors within a gene is highly associated with its gene expression levels $[21,22]$. There are several DNase I hypersensitivity sites within the Il4/Ill3 locus. Gene deletion experiments have shown that among those elements, conserved non-coding sequence 1 (CNS-1) and DNase I hypersensitivity site $\mathrm{V}_{\mathrm{A}}$ are important for IL-4 production [23-25]. Two DNase I hypersensitivity sites within intron 2 of the Il4 gene, HSII and HSIII, are also associated with IL-4 production. HSII has been suggested important in IL-4 production in mast cells [26]. We utilized a real-time PCR based assay (REA, restriction endonuclease accessibility assay) for measuring the "openness" of the sites in the Il4 locus [27]. In Th2 cells, all four Il4 locus sites, CNS-1, $\mathrm{V}_{\mathrm{A}}$, HSII and HSIII, show strikingly increased accessibility compared to that in Th1 cells. Cells primed under Th1 conditions that were infected with a GATA3-RV show increased accessibility in the Il4 locus at the CNS-1 and $\mathrm{V}_{\mathrm{A}}$ sites, but not at HSII and HSIII sites. By contrast, cells primed under Th1 cells infected with the STAT5A1*6-RV had enhanced accessibility at the HSII and HSIII sites but not at the CNS- 1 and $\mathrm{V}_{\mathrm{A}}$ sites. Cells primed under Th1 conditions that expressed both GATA-3 and STAT5A $1 * 6$ opened all four sites to a similar degree to that in Th2 cells.

Furthermore, it has been shown that GATA-3 is bound to the $\mathrm{V}_{\mathrm{A}}$ site in Th2 cells [28]. Using chromatin immunoprecipitation (ChIP), we found that Stat5 is bound to both the HSII and HSIII sites in Th2 but not Th1 cells $[12,13]$. These results thus indicate that GATA-3 and Stat5 directly bind to and modulate the Il4 locus at different sites.

\section{Gfi-1 and Th2 expansion}

We have reported that growth factor independent-1 (Gfi-1) was induced by IL-4 signaling in activated T cells through Stat6 [29]. TCR signaling alone can induce Gfi-1; however, the induction of Gfi-1 expression was substantially prolonged when cells were cultured in the presence of IL-4. Gfi-1 expression levels fell after several days of $\mathrm{T}$ cell stimulation under all conditions. Enforced expression of Gfi-1 by retroviral infection of Th2 cells resulted in sustained cell expansion when cells were cultured in IL-2-containing medium; no such effect was noted in Gfi-1-RV-infected Th1 cells. Since GATA-3 expression is

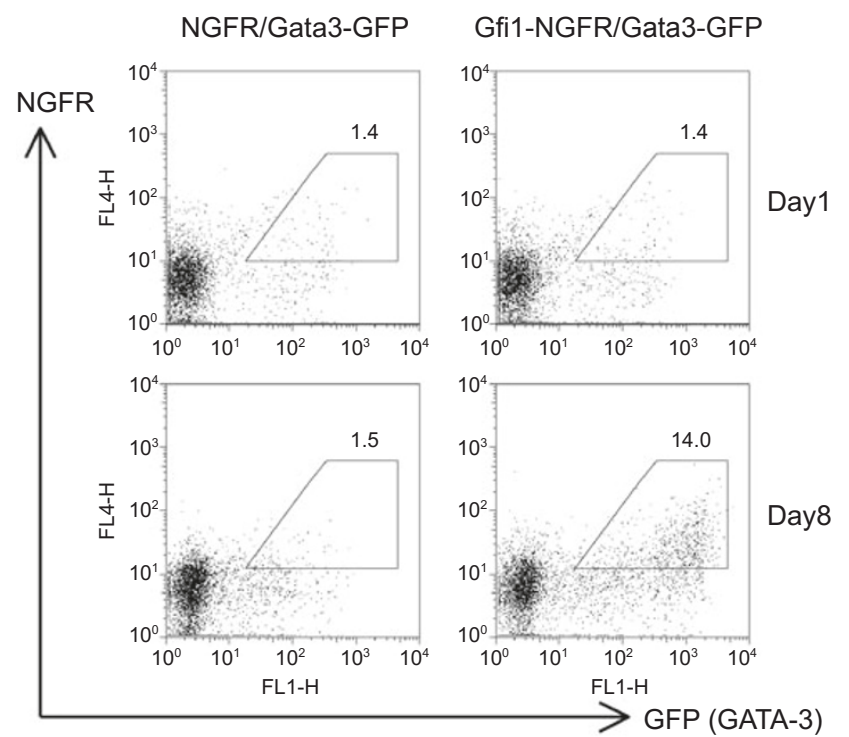

Figure 2 GATA-3 is a cofactor of Gfi-1 in promoting cell expansion. Naïve Stat $6^{-/}$CD4 T cells were activated and co-infected under ThN conditions with GATA-3-GFP-RV and Gfi-1-NGFR-RV or control NGFR-RV. Staining for NGFR expression was carried out on $\mathrm{d} 1$ and d 8 of culture in IL-2, and NGFR and GFP expression are shown. Numbers in the plots indicate the percentage of $\mathrm{NGFR}^{+} \mathrm{GFP}^{+}$cells. 
strikingly different between Th1 and Th2 cells, we considered the possibility that it might determine the differential responsiveness of the two cell types to Gfi-1. To study this, cells primed under Th1 conditions were co-infected with GATA-3-RV and Gfi-1-RV. Stat $6^{-/-}$CD4 T cells that expressed both transcription factors displayed a striking growth advantage (Figure 2). Interestingly, although Gfi-1 was originally cloned from an IL-2-independent cell line, leading to its designation as "growth factor independent" [30], in Th2 cells, IL-2 is required for their proliferation. Indeed, one interpretation of its function is that it prolongs the period during which the cells show responsiveness to IL-2.

The observation that enforced Gfi- 1 expression caused enhanced Th2 cell expansion does not necessarily mean Gfi-1 plays a role in Th2 cell expansion under physiological conditions. Germline Gfil knockout mice had previously been made by two groups $[31,32]$; many abnormalities have been reported, including neutropenia, a $\mathrm{T}$ cell development defect, a hematopoietic stem cell defect as well as defects in dendritic cell development and function [31-36]. On the other hand, because of the profound defect in $T$ cell development $[32,33]$, it was not possible to analyze the role played by Gfi-1 in the differentiation and/or growth of mature CD4 T cells. To test the physiological function of Gfi-1 in peripheral T cells, we made a Gfil-conditional knockout mice strain using Cre-loxP system. Our homozygous floxed (allele flanked by loxP sites) Gfi-1 mice $\left(G f i I^{\mathrm{f} / \mathrm{fl}}\right)$, in which the exons encoding 4 zinc-finger domains were flanked by loxP sites, appeared grossly normal without obvious defects (unpublished observations). Most importantly, those mice have normal-sized thymuses with CD4/CD8 profiles similar to those of wild-type mice. CD4 T cells were purified from lymph nodes of $G f I^{\mathrm{fl} / \mathrm{fl}}$ mice and primed under Th2 conditions; Cre was introduced by RV infection. The Cre-RV carries a GFP marker. After priming, the relative capacity of the Gfi- $1^{+}$and $\mathrm{Gfi}-1^{-}$cells to expand in IL-2 was determined by the monitoring ratio of $\mathrm{GFP}^{+} / \mathrm{GFP}^{-}$cells. While infection with Cre-RV has very little effect on Th2 cells from wild type mice, infection of $G f i 1^{\mathrm{t} / \mathrm{fl}}$ cells with the Cre-RV, resulting the deletion of $G$ Gil, significantly reduced the rate of cell expansion (unpublished observations).

To further test whether Gfi-1 is also important for in vivo Th2 responses, $G f i 1^{\mathrm{t} / \mathrm{ll}}$ mice were bred to CD4-Cre transgenic mice [37]. Unlike the germline Gfil knockout, the G $f i 1^{\mathrm{f} / \mathrm{f}} \mathrm{CD} 4 \mathrm{Cre}$ mice have normal CD4 T cell development with slightly increased percentage of single positive CD8 cells in the thymus (unpublished observations). $G$ fi $i{ }^{\mathrm{f} / \mathrm{fl}} \mathrm{CD}$ 4Cre mice were infected with Schistosoma mansoni. Eight weeks later, cytokine production of mesenteric lymph node cells was measured after ex vivo stimulation. Mesenteric lymph node CD4 cells from Gfi ${ }^{\mathrm{t} / \mathrm{fl}} \mathrm{CD} 4 \mathrm{Cre}$ ( $G f i 1$-deficient CD4 cells) made substantially less IL-4 than did cells from control infected mice. In addition, the proportion of cells expressing large amounts of GATA-3, as determined by intracellular staining was less than that among the control Gfil-sufficient group. In vitro stimulation of such cells with SEA for 5 days significantly increased the percentage of GATA-3 ${ }^{\text {hi }}$ cells in both groups; however, there were still fewer GATA- $3^{\text {hi }}$ cells in the Gfil-deficient population. Furthermore, after another 3 days culture in IL-2 medium, most of the cells in control group were GATA- $3^{\text {hi }}$, whereas more than $50 \%$ of the cells in Gfil-deficient group were GATA- $3^{\text {lo }}$ (unpublished observations).

We conclude that forced expression of Gfi- 1 results in a proliferative advantage for GATA- $3^{\text {hi }}$ cells in IL-2, leading to the dominance of the population of Gfi- $1^{+}$GATA $-3^{\text {hi }}$ cells. Conversely, deletion of Gfil impairs normal Th2 cells expansion both in vitro and in vivo, resulting in a lower percentage of GATA- $3^{\text {hi }}$ cells. These results imply that Gfi- 1 plays an important physiologic role in Th2 cell expansion and that by acting only on Th2 cells helps to explain how these cells may come to dominate the population of cells responding to given stimuli.

\section{The central role of GATA-3 in reinforcing Th2 re- sponses}

GATA-3 is differentially expressed by Th1 and Th 2 cells $[19,20]$. Enforced expression of GATA-3, by retroviral infection, induced Th2 responses independent of IL-4/Stat6 signaling [38]. Introduction of anti-sense GATA-3 or of a dominant negative form of GATA-3 limited Th2 responses $[20,39]$. Our data also showed that GATA-3 can induce IL-4 production and that it is required for Gfi- 1 mediated cell growth $[13,29]$. Introducing constitutively active STAT5 (STAT5A1*6) can also drive an IL-4 independent Th2 response and it does so without up-regulating GATA-3 expression. This raises the question of whether GATA-3 is required for Th2 differentiation, especially when it occurs under IL-4 independent circumstances.

The role of GATA-3 in Th2 differentiation has not been carefully verified by gene deletion experiments. Part of the difficulty in doing so is that deleting Gata3 results in embryonic lethality [40]. Furthermore, GATA-3 is involved in multiple stages of CD4 T cell development. First, GATA3-deficient hematopoietic stem cells fail to generate T cells in transfer experiments [41]. Second, using conditional Gata3 knockout mice, it was observed that deletion in CD4/CD8 double-negative thymocytes blocked T cell development at the DN3 stage [42]. Third, deleting Gata3 at the double positive $\left(\mathrm{CD} 4^{+} \mathrm{CD} 8^{+}\right)$stage results in a severe impairments of the development of CD4 single positive $\mathrm{T}$ 
cells, although development of CD8 single positive T cells appears normal $[42,43]$.

We prepared a conditional Gata3 knockout by flanking exon 4 of the Gata3 gene with two loxP sites [43]. Exon 4 encodes the N-terminal of the zinc-fingers of GATA-3 and its deletion also introduces a reading frame shift so that the $\mathrm{C}$-terminal portion of the molecule, including the C-terminal zinc-fingers, will not be expressed.

These homozygous Gata 3 floxed $\left(\right.$ Gata $\left.{ }^{\mathrm{f} / \mathrm{fl}}\right)$ mice appear to be normal, as expected. CD4 T cells were purified from lymph nodes of Gata $^{\mathrm{ft} / \mathrm{fl}}$ mice; Cre was introduced by retroviral infection during the initial priming of naïve CD4 T cells under Th2 conditions or after the Th2 phenotype had been established. Deletion of Gata3 during the initiation of Th2 differentiation greatly reduced the capacity of the cells to produce IL-4 upon re-stimulation [43]. However, if Gata 3 was deleted in established Th2 cells, only a modest decrease was noted in the percentage of IL-4 producing cells, accompanied by a 2 3-fold reduction in MFI. Il4 chromatin accessibility tested by REA was dramatically decreased at all the sites tested including HSII, HSIII in intron 2 and $\mathrm{V}_{\mathrm{A}}$ at 3' of Il4 gene when Gata3 was deleted during initial Th2 priming (unpublished observations). However, only accessibility at $\mathrm{V}_{\mathrm{A}}$ site of the Il4 gene, but not at the HSII and HSIII sites, was lost when Gata3 was deleted in the differentiated Th2 cells. This is consistent with reported data indicating that GATA-3 is bound to the $\mathrm{V}_{\mathrm{A}}$ site in Th2 cells [28]. Thus, GATA-3 is required for promoting the opening of Il4 chromatin during the early Th2 differentiation but is dispensable for maintaining the accessibility of the Il4 gene when the Th2 phenotype has been well established. However, the accessibility of Il4 gene at the $\mathrm{V}_{\mathrm{A}}$ site continues to depend on the presence of GATA-3, implying that the capacity of GATA-3 to enhance IL-4 production in differentiated Th2 cells depends on its action on the 3' enhancer, which contains $\mathrm{V}_{\mathrm{A}}$.

The limited effect of Gata3 deletion on IL-4 production in established Th2 cells was confirmed by the analysis of Th2 cells that appeared in mice infected with Nippostrongylus brasiliensis [43]. In such experiments, the deletion of Gata3 by infection with a Cre-RV had no effect on the percentage of IL-4-producing cells but reduced the MFI of these cells by $2 \sim 3$ fold. Interestingly, although the IL-4 production is modestly affected, IL-5 and IL-13 production by those cells was totally lost upon Gata3 deletion. It has been shown that there are GATA-3-binding sites in the IlI and Ill 3 promoters but not in the Il4 promoter [4449]. Thus, GATA-3 is indispensable for IL-5 and IL-13 production in Th2 cells.

Introduction of STAT $5 \mathrm{~A} 1 * 6$ into cells during initial stimulation can induce Th2 differentiation independent of IL-4/Stat6 signaling and without up-regulating GATA-3 expression [13]. However, in view of the distinctive role of Stat5 and GATA-3 in the activation of the Il4 gene, it was important to determine whether GATA-3 was completely dispensable for Th2 priming in cells expressing STAT $5 \mathrm{~A} 1 * 6$. We examined the role of GATA-3 in a co-infection experiment, in which a STAT5A $1 * 6-N G F R-R V$ and a Cre-GFP-RV were introduced into Gata $3^{\mathrm{f} / \mathrm{fl}} \mathrm{CD} 4 \mathrm{~T}$ cells primed under Th1 conditions. As expected, in $\mathrm{GFP}^{-}$group, STAT 5 A $1 * 6$ expression induced substantial IL-4 production compared to uninfected cells. However, within $\mathrm{GFP}^{+}$ group, in which Gata3 was presumably deleted by Cre, STAT5A1*6 failed to induce IL-4 production [43]. Thus, although the GATA-3 levels are very low in STAT5A1*6driven (IL-4 independent) Th2 differentiation, such low level expression of GATA-3 is critical for generating IL4-producing Th2 cells.

Early IL-4 production is associated with IL-4-independent GATA-3 up-regulation and Gata3 deletion in naïve CD4 $\mathrm{T}$ cells totally abrogates the early IL-4 production [16]. These data suggest that GATA-3 is important for IL4-independent IL-4 production and Th2 differentiation as well as for IL-4-dependent Th2 differentiation.

Gfi-1 induces the expansion of GATA-3-expressing cells, suggesting that $\mathrm{Th} 2$ cells are selected to grow as a result of IL-4 stimulation. Consistent with this idea, the Gata3-deleted "Th2" cells expanded poorly [43]. In a BrdU uptake experiment, the Gata3-deleted cells had a substantially diminished proportion of BrdU positive cells. We also found that in vitro deletion of Gata3 floxed alleles by Cre-RV was incomplete; thus, within the sorted $\mathrm{GFP}^{+}\left(\mathrm{Cre}^{+}\right)$ cells, there were a percentage of cells $(2 \sim 5 \%)$ that failed to undergo Gata3 deletion; in these cells, the floxed alleles can be detected by specific real-time PCR. Those cells preferentially expanded in the culture. Indeed, after three additional rounds of Th 2 priming, more than $80 \%$ of the cells carried floxed alleles, implying that the limited number of cells that had failed to delete Gata 3 alleles, had a marked growth advantage over those cells that completely deleted Gata3. These data also suggest that GATA-3 plays an important role in Th2 cell expansion.

To obtain a cell population with complete Gata3 deletion, single cell cloning under Th2 conditions was carried out after one round of Th2 priming and Cre-RV infection

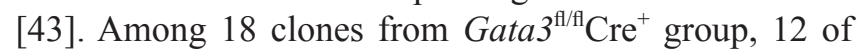
them had deleted Gata3 $\left(\right.$ Gata3 $\left.^{-1-}\right)$. Each of those 12 Gata3 $^{-}$ - clones produced a certain amount of IL-4 although the expression levels were generally lower than that of the clones from $\mathrm{Fata}^{\mathrm{f} / /+} \mathrm{Cre}^{+}$clones. Chromatin accessibility of the Il4 locus at the HSII and HSIII sites was similar to that of normal Th2 cells, but accessibility at the $\mathrm{V}_{\mathrm{A}}$ site was greatly diminished (unpublished observations). No detectable IL-5 and IL-13 were noted in those Gata3"-- "Th2" 
clones. Strikingly, all the Gata3 $3^{--~ " T h 2 " ~ c l o n e s ~ w e r e ~ a b l e ~}$ to produce a large amount of IFN $\gamma$ [43]. The majority of the IL-4-producing cells from those clones were also IFN $\gamma$ producing cells. Real-time PCR showed that the expression of c-Maf [50], a transcription factor critical for IL-4 production, was not reduced but the T-bet expression was greatly enhanced in the Gata3"- "Th2" clones (Figure 3). Re-introduction of GATA-3 by retroviral infection restored the capability of the Gata3 $3^{-/-}$"Th2" clones to produce IL-5 and IL-13; IL-4 production was also enhanced by 2 3 fold; however, IFN $\gamma$ production was not inhibited (unpublished observations).

When Gata $^{\text {ft/fl }}$ mice were bred to CD4-Cre, a severe defect in CD4 single positive T cell development was found, consistent with previous reports $[42,43]$. Of those small percentage of CD4 T cells in the peripheral, the majority display a memory-like phenotype [43]. Thus, CD4-Cre is not a suitable tool to study the effect of Gata3 deletion in mature CD4 T cells. To test whether GATA-3 is indeed important for in vivo Th2 responses, OX40-Cre was used to delete Gata3 gene in vivo. Unlike CD4-Cre, OX40-Cre is not expressed in most thymocytes; thus, CD4 $\mathrm{T}$ cell development in Gata $^{\mathrm{H} / \mathrm{fl}} \mathrm{OX} 40$-Cre mice was normal

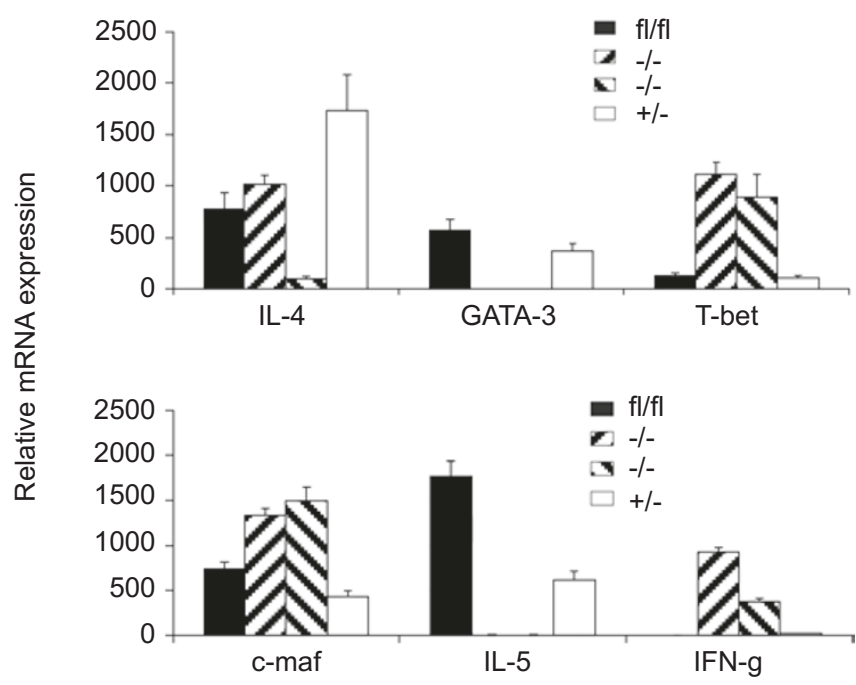

Figure 3 Enhanced expression of Th1 factors in Gata $^{-/-}$"Th2" clones. Naïve CD4 T cells were activated and infected with Cre-GFPRV under Th2 (IL-4, anti-IFN $\gamma$ and anti-IL-12) conditions. $\mathrm{Cre}^{+} \mathrm{Gata}^{\mathrm{fl} /}$ ${ }^{\mathrm{fl}}$ and $\mathrm{Cre}^{+}$Gata $^{\mathrm{fl} /+}$ cells were sorted based on GFP expression and single cell cloning was carried out. The clones were genotyped after eight weeks of priming under Th2 conditions. The mRNA levels of transcription factors and cytokines in four different clones were measured by real-time PCR after PMA/ionomycin stimulation for two hours. Fl/fl: a $\mathrm{Cre}^{+}$Gata $^{\mathrm{f} / \mathrm{fl}}$ clone in which neither of the floxed Gata3 alleles were deleted; -/-: a Gata3 deleted clone; +/-: a $\mathrm{Cre}^{+}$Gata $3^{\mathrm{fl} /+}$ clone in which the floxed Gata3 allele was deleted.
[43]. CD4 T cells in the peripheral from Gata $^{\mathrm{f} / \mathrm{f}} \mathrm{OX} 40-\mathrm{Cre}$ mice were mostly naïve, as in wild type mice. Infection of Gata $3^{\mathrm{fl} / \mathrm{I}} \mathrm{OX} 40-\mathrm{Cre}$ mice with Nippostrongylus brasiliensis demonstrated that GATA-3 is critical for in vivo Th2 responses. In the Gata $3^{\mathrm{f} / \mathrm{fl}}$ mice, N. brasiliensis worms were cleared after 10 days of infection and a high percentage of IL-4/IL-13-producing CD4 cells was detected in mesenteric lymph nodes and serum IgE levels were elevated. By contrast, in the Gata $3^{\mathrm{t} / \mathrm{fl}} \mathrm{OX} 40$-Cre mice, there were high worm burden at 10 days of infection, very few IL-4/IL13-producing CD4 cells detected and serum IgE levels remained low. Significant numbers of IFN $\gamma$-producing cells were found in Gata $^{\mathrm{f} / / \mathrm{f}} \mathrm{OX} 40-\mathrm{Cre}$ mice after infection [43]. Thus, although the Th2 response to helminth infection is IL-4/Stat6 independent [51-54], it is GATA-3 dependent.

\section{Conclusion}

GATA-3 plays a central role in Th2 responses both in vitro and in vivo [43]. There are three different mechanisms through which GATA-3 can promote Th2 responses, in collaborating with other transcription factors (Figure 4).

First, GATA-3 is important for Th2 cytokine production. GATA-3 can be initially up-regulated through IL-4-independent pathways [16]. GATA-3 collaborates with active Stat5 in initial as well as late IL-4 production $[12,13$, $16,43]$. These two factors act on different part of the Il4 locus. Initial IL-4 production causes further up-regulation of GATA-3. Although GATA-3 is important for initiating

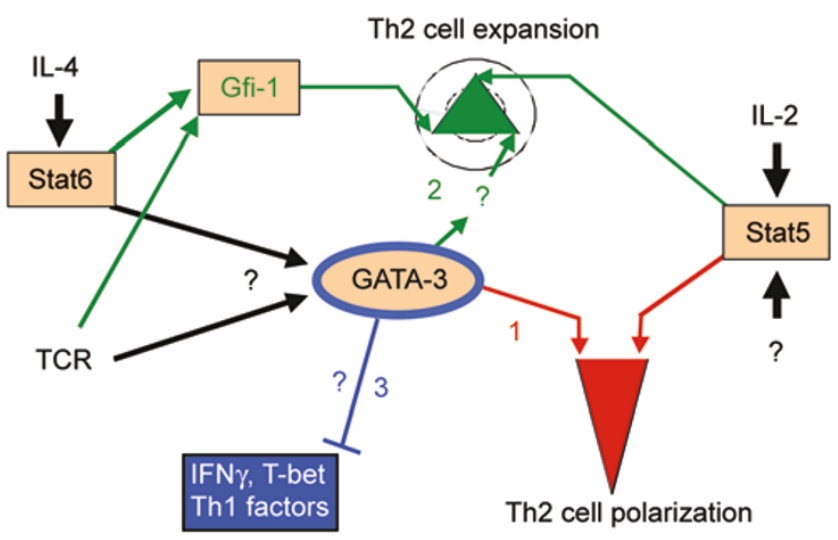

Figure 4 GATA-3 promotes Th2 responses through three different mechanisms. 1, GATA-3, induced by TCR and/or IL-4 signaling, collaborates with Stat5, activated by IL-2 and/or other cytokines, in Th2 cytokine production (red). 2, Gfi-1, induced by TCR and IL-4 signaling, selectively enhances the growth rate of GATA- $3^{\text {hi }}$ cells in response to cytokines such as IL-2 (green). 3, GATA-3, even expressed at basal levels, suppresses the expression of Th1 factors like IFN $\gamma$ and T-bet (blue). ?: unknown factors or mechanisms. 
the Th2 polarization it is less critical for maintaining the accessibility of Il4. However, GATA-3 is indispensable for IL-5 and IL-13 production even in fully differentiated Th2 cells.

Second, GATA- $3^{\text {hi }}$ cells can be selected to expand by Gfi-1, an IL-4/Stat6 inducible gene product [29, 43]. In the absence of Gfi- 1 , the expansion of GATA- $3^{\text {hi }}$ cells is impaired resulting in diminished $\mathrm{Th} 2$ responses.

Third, GATA-3, even when expressed at basal levels, inhibits Th1 responses [43]. Without GATA-3, Th1 responses occur even in the absence of the Th1 inducing factor, IL12 and IFN $\gamma$. The inhibition of Th1 responses by GATA-3 presumably is through inhibiting the expression of T-bet.

Therefore, a certain level of GATA-3 expression is critical for CD4 $\mathrm{T}$ cells to maintain the non-polarized phenotype. During Th1 responses, GATA-3 expression is down-regulated, possibly relieving repression of T-bet expression. T-bet can then promote Th1 responses through three mechanisms similar to that of GATA-3 in Th2 responses: induction of Th1 cytokine production, promotion of the selective growth of Th1 cells and inhibition of Th2 responses. T-bet induces the production of IFN $\gamma$, which is a potent inducer of T-bet [4, 6]; T-bet up-regulates IL-12R $\beta 2$ so that cells can be selected to grow in response to IL-12 [7]; T-bet may down-regulate GATA-3 function either by regulating its expression or by inhibiting its activity $[4,55]$. Thus, controlling of the GATA-3 expression is the central event in initiating $\mathrm{Th} 2$ as well as Th1 responses.

\section{Reference}

1 Mowen KA, Glimcher LH. Signaling pathways in Th2 development. Immunol Rev 2004; 202:203-22.

2 Murphy KM, Reiner SL. The lineage decisions of helper T cells. Nat Rev Immunol 2002; 2:933-44.

3 Laouar Y, Sutterwala FS, Gorelik L, Flavell RA. Transforming growth factor-beta controls $\mathrm{T}$ helper type 1 cell development through regulation of natural killer cell interferon-gamma. Nat Immunol 2005; 6:600-7.

4 Szabo SJ, Kim ST, Costa GL, et al. A novel transcription factor, T-bet, directs Th1 lineage commitment. Cell 2000; 100:65569.

5 Szabo SJ, Sullivan BM, Stemmann C, et al. Distinct effects of T-bet in Th1 lineage commitment and IFNgamma production in CD4 and CD8 T cells. Science 2002; 295:338-42.

6 Lighvani AA, Frucht DM, Jankovic D, et al. T-bet is rapidly induced by interferon-gamma in lymphoid and myeloid cells. Proc Natl Acad Sci USA 2001; 98:15137-42.

7 Afkarian M, Sedy JR, Yang J, et al. T-bet is a STAT1-induced regulator of IL-12R expression in naive CD4+ T cells. Nat Immunol 2002; 3:549-57.

8 Mullen AC, High FA, Hutchins AS, et al. Role of T-bet in commitment of Th1 cells before IL-12-dependent selection. Science 2001; 292:1907-10

9 Nelms K, Keegan AD, Zamorano J, Ryan JJ, Paul WE. The IL-4 receptor: Signaling mechanisms and biologic functions. Annu Rev Immunol 1999; 17:701-38.

10 Kaplan MH, Schindler U, Smiley ST, Grusby MJ. Stat6 is required for mediating responses to IL-4 and for the development of Th2 cells. Immunity 1996; 4:313-9.

11 Zhu J, Guo L, Watson CJ, Hu-Li J, Paul WE. Stat6 is necessary and sufficient for IL-4's role in Th2 differentiation and cell expansion. J Immunol 2001; 166:7276-81.

12 Cote-Sierra J, Foucras G, Guo L, et al. Interleukin 2 plays a central role in Th2 differentiation. Proc Natl Acad Sci USA 2004; 101:3880-5.

13 Zhu J, Cote-Sierra J, Guo L, Paul WE. STAT5 activation plays a critical role in Th2 differentiation. Immunity 2003; 19:739-48.

14 Le Gros G, Ben-Sasson SZ, Seder R, Finkelman FD, Paul WE. Generation of interleukin 4 (IL-4)-producing cells in vivo and in vitro: IL-2 and IL-4 are required for in vitro generation of IL-4-producing cells. J Exp Med 1990; 172:921-9.

15 Ben-Sasson SZ, Le Gros G, Conrad DH, Finkelman FD, Paul WE. IL-4 production by T cells from Naïve donors. IL-2 is required for IL-4 production. J Immunol 1990; 145:1127-36.

16 Yamane H, Zhu J, Paul WE. Independent roles for IL-2 and GATA-3 in stimulating naive CD4+ T cells to generate a Th2-inducing cytokine environment. J Exp Med 2005; 202:793-804.

17 Lin JX, Leonard WJ. The role of Stat5A and Stat5B in signaling by IL-2 family cytokines. Oncogene 2000; 19:2566-76.

18 Onishi M, Nosaka T, Misawa K, et al. Identification and characterization of a constitutively active STAT5 mutant that promotes cell proliferation. Mol Cell Biol 1998; 18:3871-9.

19 Zhang DH, Cohn L, Ray P, Bottomly K, Ray A. Transcription factor GATA-3 is differentially expressed in murine Th1 and Th2 cells and controls Th2-specific expression of the interleukin-5 gene. J Biol Chem 1997; 272:21597-603.

20 Zheng W, Flavell RA. The transcription factor GATA-3 is necessary and sufficient for Th2 cytokine gene expression in $\mathrm{CD} 4^{+} \mathrm{T}$ cells. Cell 1997; 89:587-96.

21 Agarwal S, Rao A. Modulation of chromatin structure regulates cytokine gene expression during $\mathrm{T}$ cell differentiation. Immunity 1998; 9:765-75.

22 Guo L, Hu-Li J, Zhu J, et al. In Th2 cells the Il4 gene has a series of accessibility states associated with distinctive probabilities of IL-4 production. Proc Natl Acad Sci USA. 2002; 99:10623-8.

23 Loots GG, Locksley RM, Blankespoor CM, et al. Identification of a coordinate regulator of interleukins 4,13 , and 5 by crossspecies sequence comparisons. Science 2000; 288:136-40.

24 Mohrs M, Blankespoor CM, Wang ZE, et al. Deletion of a coordinate regulator of type 2 cytokine expression in mice. Nat Immunol 2001; 2:842-47.

25 Solymar DC, Agarwal S, Bassing CH, Alt FW, Rao A. A 3' enhancer in the IL-4 gene regulates cytokine production by Th2 cells and mast cells. Immunity 2002; 17:41-50.

26 Hural JA, Kwan M, Henkel G, Hock MB, Brown MA. An intron transcriptional enhancer element regulates $I l 4$ gene locus accessibility in mast cells. J Immunol 2000; 165:3239-49.

27 Guo L, Hu-Li J, Paul WE. Probabilistic regulation of IL-4 production in Th2 cells: accessibility at the Il4 locus. Immunity 2004; 20:193-203.

28 Agarwal S, Avni O, Rao A. Cell-type-restricted binding of the transcription factor NFAT to a distal IL-4 enhancer in vivo. Immunity $2000 ; \mathbf{1 2}: 643-52$. 
29 Zhu J, Guo L, Min B, et al. Growth factor independent-1 induced by IL-4 regulates Th2 cell proliferation. Immunity $2002 ; 16: 733-$ 44.

30 Gilks CB, Bear SE, Grimes HL, Tsichlis PN. Progression of interleukin-2 (IL-2)-dependent rat $\mathrm{T}$ cell lymphoma lines to IL-2-independent growth following activation of a gene (Gfil) encoding a novel zinc finger protein. Mol Cell Biol 1993; 13:1759-68.

31 Karsunky H, Zeng H, Schmidt T, et al. Inflammatory reactions and severe neutropenia in mice lacking the transcriptional repressor Gfi-1. Nat Genet 2002; 30:295-300.

32 Hock H, Hamblen MJ, Rooke HM, et al. Intrinsic requirement for zinc finger transcription factor Gfi-1 in neutrophil differentiation. Immunity 2003; 18:109-20.

33 Yucel R, Karsunky H, Klein-Hitpass L, Moroy T. The transcriptional repressor Gfi-1 affects development of early, uncommitted $\mathrm{c}-\mathrm{Kit}^{+} \mathrm{T}$ cell progenitors and CD4/CD8 lineage decision in the thymus. J Exp Med 2003; 197:831-44.

34 Hock H, Hamblen MJ, Rooke HM, et al. Gfi-1 restricts proliferation and preserves functional integrity of haematopoietic stem cells. Nature 2004; 431:1002-7.

35 Zeng H, Yucel R, Kosan C, Klein-Hitpass L, Moroy T. Transcription factor Gfil regulates self-renewal and engraftment of hematopoietic stem cells. EMBO J 2004; 23:4116-25.

36 Rathinam C, Geffers R, Yucel R, et al. The transcriptional repressor Gfi-1 controls STAT3-dependent dendritic cell development and function. Immunity 2005; 22:717-28.

37 Lee PP, Fitzpatrick DR, Beard C, et al. A critical role for Dnmt1 and DNA methylation in $\mathrm{T}$ cell development, function, and survival. Immunity 2001; 15:763-74.

38 Ouyang W, Ranganath SH, Weindel K, et al. Inhibition of Th1 development mediated by GATA-3 through an IL-4-independent mechanism. Immunity 1998; 9:745-55.

39 Zhang DH, Yang L, Cohn L, et al. Inhibition of allergic inflammation in a murine model of asthma by expression of a dominant-negative mutant of GATA-3. Immunity 1999; 11:473-82.

40 Pandolfi PP, Roth ME, Karis A, et al. Targeted disruption of the Gata 3 gene causes severe abnormalities in the nervous system and in fetal liver haematopoiesis. Nat Genet 1995; 11:40-4.

41 Ting CN, Olson MC, Barton KP, Leiden JM. Transcription factor GATA-3 is required for development of the T-cell lineage. Nature 1996; 384:474-8.

42 Pai SY, Truitt ML, Ting CN, et al. Critical roles for transcription factor GATA-3 in thymocyte development. Immunity 2003;
19:863-75.

43 Zhu J, Min B, Hu-Li J, et al. Conditional deletion of Gata3 shows its essential function in Th1-Th2 responses. Nat Immunol 2004; 5:1157-65.

44 Siegel MD, Zhang DH, Ray P, Ray A. Activation of the interleukin-5 promoter by cAMP in murine EL-4 cells requires the GATA-3 and CLE0 elements. J Biol Chem 1995; 270:2454855.

45 Zhang DH, Yang L, Ray A. Differential responsiveness of the Il5 and Il4 genes to transcription factor GATA-3. J. Immunol. 1998; 161:3817-21.

46 Klein-Hessling S, Jha MK, Santner-Nanan B, et al. Protein kinase A regulates GATA-3-dependent activation of $I l 5$ gene expression in Th2 cells. J. Immunol. 2003; 170:2956-61.

47 Kishikawa H, Sun J, Choi A, Miaw SC, Ho IC. The cell type-specific expression of the murine 1113 gene is regulated by GATA-3. J Immunol 2001; 167:4414-20.

48 Lavenu-Bombled C, Trainor CD, Makeh I, Romeo PH, MaxAudit I. Interleukin-13 gene expression is regulated by GATA-3 in T cells: role of a critical association of a GATA and two GATG motifs. J Biol Chem 2002; 277:18313-21.

49 Yamashita M, Ukai-Tadenuma M, Kimura M, et al. Identification of a conserved GATA3 response element upstream proximal from the interleukin-13 gene locus. J Biol Chem 2002; 277:42399408.

50 Kim JI, Ho IC, Grusby MJ, Glimcher LH. The transcription factor c-Maf controls the production of interleukin- 4 but not other Th2 cytokines. Immunity 1999; 10:745-51.

51 Noben-Trauth N, Shultz LD, Brombacher F, et al. An interleukin 4 (IL-4)-independent pathway for $\mathrm{CD}^{+} \mathrm{T}$ cell IL-4 production is revealed in IL-4 receptor-deficient mice. Proc Natl Acad Sci USA 1997; 94:10838-43.

52 Jankovic D, Kullberg MC, Noben-Trauth N, et al. Single cell analysis reveals that IL-4 receptor/STAT6 signaling is not required for the in vivo or in vitro development of $\mathrm{CD} 4+$ lymphocytes with a Th2 cytokine profile. J Immunol 2000; 164:3047-55.

53 Finkelman FD, Morris SC, Orekhova T, et al. STAT6 regulation of in vivo IL-4 responses. J Immunol 2000; 164:2303-10.

54 Mohrs M, Shinkai K, Mohrs K, Locksley RM. Analysis of type 2 immunity in vivo with a bicistronic IL-4 reporter. Immunity 2001; 15:303-11.

55 Hwang ES, Szabo SJ, Schwartzberg PL, Glimcher LH. T helper cell fate specified by kinase-mediated interaction of T-bet with GATA-3. Science 2005; 307:430-3. 